\title{
Treatment with Enterococcus faecalis CECT7121 Is Not Effective as Therapy in Mice with an Established Allergy Status
}

\author{
Ailén M. Díaz¹, Matías A. Molina', Guillermo G. Nuñez¹, Ana C. Mourelle¹, Mónica D. Sparo², \\ Marcela A. Manghi ${ }^{1}$, Marisa S. Castro ${ }^{*}$ \\ ${ }^{1}$ Facultad de Farmacia y Bioquímica, Universidad de Buenos Aires, Instituto de Estudios de la Inmunidad Humoral "Prof. Dr. R.A. \\ Margni” (IDEHU, CONICET-UBA), Buenos Aires, Argentina \\ ${ }^{2}$ Cátedra Microbiología y Parasitología (CUDEMyP), Facultad de Ciencias Médicas, Universidad Nacional de La Plata, La Plata, Argentina \\ Email: *mcastro@ffyb.uba.ar
}

How to cite this paper: Díaz, A.M., Molina, M.A., Nuñez, G.G., Mourelle, A.C., Sparo, M.D., Manghi, M.A. and Castro, M.S. (2017) Treatment with Enterococcus faecalis CECT7121 Is Not Effective as Therapy in Mice with an Established Allergy Status. Advances in Microbiology, 7, 696-706.

https://doi.org/10.4236/aim.2017.710055

Received: August 12, 2017

Accepted: October 28, 2017

Published: October 31, 2017

Copyright $\odot 2017$ by authors and Scientific Research Publishing Inc. This work is licensed under the Creative Commons Attribution-NonCommercial International License (CC BY-NC 4.0). http://creativecommons.org/licenses/by-nc/4.0/ (c) (i) \& Open Access

\begin{abstract}
In allergies, an unbalanced immune response towards a $\mathrm{T}$ helper (Th) 2 profile with high levels of Immunoglobulin (Ig) E is produced. We have demonstrated that the pre-administration of Enterococcus faecalis CECT7121 prevents the development of allergy in ovalbumin-immunized mice. In this work, we evaluated whether this bacterium can also revert an established allergic status. Mice were immunized with ovalbumin (OVA) and after that, were inoculated with an $E$. faecalis CECT7121 suspension. In immunized animals, serum specific immune response, proliferative activity of memory splenocytes, and levels of Th2 cytokines were assessed. The in vivo active cutaneous anaphylaxis test was also performed. The treatment with E. faecalis CECT7121 only increased anti-OVA IgG2a levels. No differences were observed in other specific immunological parameters. Probiotic-treatment did not prove to have any desensitizing effect on mice. These results, together with those recently published, can be concluded that this bacterium would not be appropriate for the treatment of allergic symptoms.
\end{abstract}

\section{Keywords}

Enterococcus faecalis CECT7121, Ovalbumin-Allergy Murine Model, Immunoglobulin E, Th2 Cytokines, Probiotics

\section{Introduction}

In most individuals suffering from allergic diseases, an imbalance of the immune response towards a Th2 cytokine profile is observed. This Th2 scenario induces 
the secretion of high levels of IgE, together with the recruitment of effect or cells to the site where the allergic inflammatory response is taking place [1] [2] [3] [4] [5]. On the other hand, it is known that in healthy individuals, a Th1 response is generated against allergens [6]. According to the hygiene hypothesis [5] [7] [8] [9], the exposition to microorganisms during the early stages of life could revert this Th2-skewed response, and in turn, it could induce the development of a Th1 phenotype and stimulate the activity of Treg cells. This observation has led to the experimental use of microorganisms to prevent or inhibit allergic diseases, being the probiotics, the most promising tool for this purpose [10]. There are prominent studies about probiotic pre-treatment which are successfully employed in allergies in different models. One of the beneficial effects that have been reported is a reduction in the production of serum-specific IgE in mice [11] [12] [13]. Probiotic bacteria can induce immune regulation or immune tolerance in allergic diseases as it had been demonstrated in a $\beta$-lactoglobulin-induced intestinal anaphylaxis in a murine model of food allergy where the oral administration of Clostridium butyricum CGMCC0313-1 ameliorated intestinal anaphylaxis symptoms and shifted the immune balance towards Th1 and Treg, with significantly increased Foxp3/Ror $\gamma t$ and Foxp3/Gata ratios and a significantly decreased Gata3/Tbet ratio [14]. Furthermore, there are other studies that show that the probiotic administration induces an improvement on allergic symptoms. Yang et al. have observed that the treatment for two weeks with Bifidobacterium infantis during OVA sensitization attenuated the serum specific IgE and IgG1 secretion as well as reduced Th2-type cytokines in spleen cell supernatants and, after challenge with OVA, probiotic-treated mice showed lower allergic reaction measured as diarrhea than sensitized-mice [15].

Enterococcus faecalis strains are frequently isolated from food products, and certain strains have been used as cheese starter and food products in the market worldwide [16]. Moreover, they are also employed as probiotics in many applications for human and animals [17]. We have previously worked with a nonpathogenic strain, Enterococcus faecalis CECT7121, isolated from natural corn silage [18]. We have evidenced that this strain implants and remains in the intestinal mucosa of BALB/c mice, stimulating the immune system [19] [20]. We have also demonstrated the adjuvant effect of E. faecalis CECT7121 and its broad pro-Th1 immunomodulatory activity, achieving beneficial effects on different experimental models [19] [21] [22] [23].

The immunomodulatory effect of E. faecalis CECT7121 has been demonstrated in an allergy model induced by the subcutaneous (s.c.) administration of Ovalbumin (OVA), where this strain was able to diminish the secretion of specific IgE and to induce an increase in the levels of specific IgG2a. This probiotic also decreased the proliferation of splenocytes and the secretion of Th2 cytokines [23]. In the latter model, we have demonstrated that the previous implantation of E. faecalis CECT7121 in the intestinal mucosa prevents the development of the allergic status. In order to perform a more insightful assessment of the immunomodulatory capacity of this probiotic, in this work, we evaluated 
whether this probiotic can also revert an established allergic status. Considering that probiotic immunomodulatory capacity is broadly assessed as pre-treatment in allergy models, but there is little information about probiotic post-treatment in an established allergy model, in this work we evaluated whether E. faecalis CECT7121 can also revert an established allergic status. For that reason, OVA-sensitized mice were treated with the probiotic by the intragastric (i.g.) route, evaluating parameters of the Th- 2 biased immune response induced by the allergen.

\section{Materials and Methods}

\subsection{Mice}

Conventional female BALB/c mice were provided by the School of Veterinary Medicine, Universidad de Buenos Aires (Argentina). All animals were housed (n = 5 mice/cage) under specific conditions according to the "Guide for the Care and Use of Laboratory Animals" (National Research Council of the National Academies, USA), with controlled air temperature $\left(20^{\circ} \mathrm{C}-22^{\circ} \mathrm{C}\right)$, humidity, and $12 \mathrm{~h}$ light/dark cycles; food and water were provided ad libitum. All mice were 6 weeks old at the beginning of the experiment.

All animal experiments were approved by the Instituto de Estudios de la Inmunidad Humoral "Prof. Dr. Ricardo A. Margni" (IDEHU, CONICET-UBA). Maximum efforts were undertaken to minimize animal suffering in all procedures.

\subsection{Immunization Protocol with OVA and i.g. Administration of Ent. faecalis CECT7121}

Mice were divided in two experimental groups: E. faecalis CECT7121/ OVA ( $\mathrm{n}=$ 6), S/OVA (saline) $(\mathrm{n}=6)$. OVA immunization [10 $\mu \mathrm{g} \mathrm{OVA}$ and $\left.1 \mathrm{mg} \mathrm{Al}(\mathrm{OH})_{3}\right]$ was performed on days 0,7 and 21 by s.c. injection $(0.2 \mathrm{ml})$ on the back of each mouse (Figure 1). An E. faecalis CECT7121 suspension $\left(3 \times 10^{8} \mathrm{CFU} / \mathrm{ml}\right.$, prepared as previously described [23] was administrated by the i.g. route $(0.2$ $\mathrm{ml} /$ mice/day) with a gavage needle on three doses: days 1,2 and 3; days 13,14 and 15 and on days 28,29 and 30 . On day 34 , serum samples were obtained and mice were euthanized by $\mathrm{CO}_{2}$ or cervical dislocation and spleens removed. Another group of mice was subjected to the Active Cutaneous Anaphylaxis Test (ACA). Control non-immunized animals were treated with saline instead OVA or E. faecalis CECT7121 ( $\mathrm{n}=3$ each).

\subsection{Evaluation of Cell Responses}

Individual spleen cell suspensions of each mice were prepared in RPMI 1640 medium (Gibco) containing fetal calf serum (Natocor), penicillin (Gibco), streptomycin (Gibco), amphotericin B (Gibco), L -glutamine (Gibco) and pyruvate (J.T. Baker) as previously described [23]. One hundred microlitres of spleen cell suspension $\left(4 \times 10^{6}\right.$ cells $\left./ \mathrm{ml}\right)$ were cultured with $100 \mu \mathrm{l}$ of ulture medium 


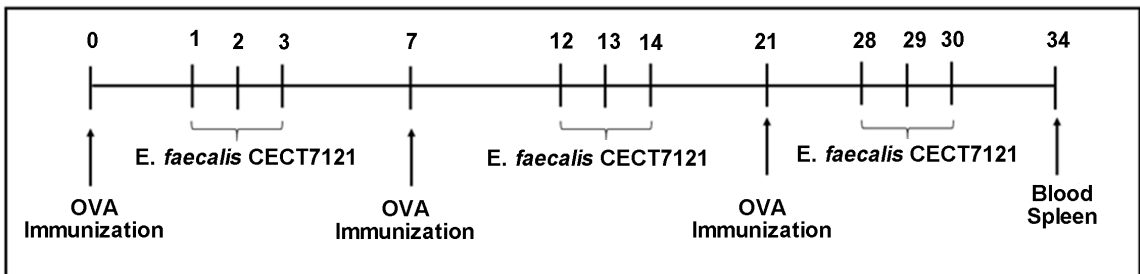

Figure 1. Immunization protocol. Mice were divided in two experimental groups: OVA/E. faecalis CECT7121 and OVA/S (saline). OVA immunization [10 $\mu$ g OVA and 1 $\mathrm{mg} \mathrm{Al}(\mathrm{OH})_{3}$ ] was performed on days 0,7 and 21 with $0.2 \mathrm{ml}$ by s.c. injection (empty arrow) and an E. faecalis CECT7121 suspension $\left(3 \times 10^{8} \mathrm{CFU} / \mathrm{ml}\right)$ was administrated with a gavage needle by the i.g. route $(0.2 \mathrm{ml} / \mathrm{mice} /$ day $)$ on days 1,2 and $3 ; 13,14$ and 15 ; and on days 28, 29 and 30. At final day 34, serum samples and splenocyte were taken, and ACA was performed. Control non-immunized animals were treated with saline instead OVA or E. faecalis CECT7121.

alone or with $100 \mu \mathrm{l}$ of OVA $\left(1 \mathrm{mg} / \mathrm{ml}\right.$; Sigma-Aldrich) in quadruplicate at $37^{\circ} \mathrm{C}$ in presence of $5 \% \mathrm{CO}_{2}$. Concanavalin A $(10 \mu \mathrm{g} / \mathrm{ml}$; Vector Labs) was used as mitogen. In each experiment, two cell cultures were performed: to assess cell proliferation and to determine the cytokine levels in supernatants. After $72 \mathrm{~h}$, supernatants were collected and stored at $-80^{\circ} \mathrm{C}$ until assayed for cytokine levels. Proliferative responses were assessed after $96 \mathrm{~h}$ of culture by $\left[{ }^{3} \mathrm{H}\right]$ thymidine (Perkin- Elmer) uptake and measured by liquid scintillation (Liquid Scintillation Analyzer 1600TR; Packard). Results were expressed as mean counts per minute \pm SEM of quadruplicate cultures.

\subsection{Levels of Cytokines and Allergen-Specific Antibodies}

Commercial kits were employed to determine levels of IL-5, IL-10 (BD Biosciences) and IL-13 (Invitrogen). Specific anti-OVA IgE and IgG antibody levels were measured by indirect ELISA in individual serum samples, employing OVA as coating antigen at $10 \mu \mathrm{g} / \mathrm{ml}$ in phosphate-buffered saline (PBS). A biotinylated anti-mouse IgE antibody (BD Biosciences) followed by an avidine-HRP solution or a HRP-conjugated anti-mouse IgG serum (Cappel) or a HRP-conjugated anti-mouse IgG1 or a HRP-conjugated anti-mouse IgG2a sera (Bethyl) were used as secondary antibodies [23]. To determine the titre of each serum sample, two-fold serial dilutions were performed. All determinations were performed in duplicate. Absorbance values were obtained after spectrophotometric reading $(450-570 \mathrm{~nm}$ ) in an ELISA plate reader (Multiskan EX; Thermo Scientific, USA). Antibody titres were calculated as the EC50 (half maximal) value obtained by a four-parameter non-linear regression curve in a log reciprocal-dilution response curve.

\subsection{Active Cutaneous Anaphylaxis}

Active Cutaneous Anaphylaxis (ACA) was induced by intradermic (i.d.) challenge with the allergen in E. faecalis CECT7121/OVA, S/OVA. Briefly, an intravenous injection of $1 \% \mathrm{w} / \mathrm{v}$ Evans Blue $(50 \mu \mathrm{l})$ was administered by the tail vein, 
and ACA was then elicited in the right ear by i.d. inoculation of $50 \mu \mathrm{l} \mathrm{OVA} \mathrm{(1}$ $\mathrm{mg} / \mathrm{ml})$. PBS was inoculated in the left ear of each animal as a control reaction. Animals were observed after $30 \mathrm{~min}$.

\subsection{Statistical Analysis}

Two separate immunization schedules were carried out. All values were presented as means with their standard errors. A linear regression of ELISA absorbance values of standards was performed. Student's $t$ test was employed to determine significance of specific antibodies results. Cell responses and cytokine levels were analyzed by the Kruskal-Wallis followed by the Dunns multiple comparisons test. In all analysis, GraphPad Prism 5.03 for Windows (GraphPad Software, USA) was used.

\section{Results and Discussion}

The aim of this work was to study the effect $E$. faecalis CECT7121 during the effector phase of the allergic response. We evaluated whether this strain could also revert Th2 parameters of the allergic response in an experimental allergy model induced by s.c. OVA administration. The parameters assessed ex vivo were secretion of specific IgE, IgG1, IgG2a antibodies and the Th2 cytokines in splenocyte culture supernatants. In addition, an in vivo of the ACA test was performed.

The development of the allergic response comprises two phases. The first one, or sensitization phase, is that $\mathrm{CD} 4^{+} \mathrm{T}$ cells become activated and differentiated into Th2 IL-4, IL-5 and IL-13-secreting cells. These cytokines would induce the secretion of IgE and IgG1 by B cells. Both basophils and mast cells have on the cell surface of the high affinity IgE receptor (FcEI), allowing this immunoglobulin to bind to the cell membrane. During a second exposure to the allergen, those IgE molecules bound to the FceI induce the receptor cross-linking leading to cell activation and the subsequent degranulation and the release of histamine, which is one of the soluble mediators responsible for the appearance of clinical symptoms of allergy. Probiotics as lactic acid bacteria have immunomodulating effects, as a reduction in the production of antigen specific serum IgE production, which is affected by IL-4 and IL-5 produced by Th2 cells [11] [12] [13] [24]. Moreover, Kalliomaki et al. have demonstrated, in a double-blind randomized placebo-controlled trial of prevention of atopic disease, that the administration of Lactobacillus rhamnosus GG prenatally to mothers and postnatally for 6 months to their infants was effective in prevention of early atopic disease in children [25].

The treatment of allergy employing probiotic microorganisms can be employed to modulate either the sensitization or the effector phase of the response. The immunomodulatory activity of probiotics and the period of time over which they have positive effects are highly dependent on the strain under study, but also with the dose, the timing of supplementation and the method of administration [26] [27] [28]. Previously, our group has demonstrated that the i.g. administration of Enterococcus faecalis CECT7121, prior to the sensitization induced 
by the administration of OVA, can prevent the establishment of the allergic response [23].

In the present work, on day 34 after the first immunization with OVA and $E$. faecalis CECT7121 treatment protocols, Th1 and Th2 parameters of the immune response were assessed. Levels of specific anti-OVA IgE and IgG1 (Th2-immunoglobulins) and IgG2a (Th1-immunoglobulin) were determined. No detectable specific immunoglobulins were found in non-immunized animals (treated or not with the probiotic; data not shown). Upon analyzing the Th2 immunoglobulin IgE, it was found that the levels of this specific anti-OVA isotype were not affected by the treatment of animals with the probiotic (Table 1). No statistical differences were found in the titres of specific IgG1 between immunized control group and those treated with the probiotic (Table 1). Contrarily, the levels of specific IgG2a were found to be higher in the group of animals immunized with OVA and treated with E. faecalis CECT7121 (Table 1). The IgG1/IgG2a ratios, therefore, were found to be significantly different between both groups of animals. The decrease in the anti-OVA IgG1/IgG2a ratios observed in the group of animals treated with the bacterium accounted for a rise in the levels of specific IgG2a. The latter finding is in line with the pro-Th1 activity already reported for E. faecalis CECT7121 [19] [21] [22].

After The proliferation rates of splenocytes obtained from both $E$. faecalis CECT7121-treated and untreated control animals did not differ from each other after the in vitro stimulation with OVA (Figure 2). Moreover, after stimulation with OVA, these splenocytes were able to secrete the Th2-related cytokines IL-5, IL-13 and the regulatory IL-10; however, no differences were found between animals immunized with OVA and those immunized and treated with $E$. faecalis CECT7121 (Figure 3) which indicates that this bacterium was not able to decrease the levels of the cytokines evaluated (Figure 3). These results are in

Table 1. Determination of specific IgE, IgG, IgG1 and IgG2a levels in serum samples by ELISA after Ova immunization of naïve $\mathrm{BALB} / \mathrm{c}$ vs. mice treated ig with $E$. faecalis CECT7121.

\begin{tabular}{|c|c|c|c|}
\hline Antibodies & Mice Group & $\begin{array}{c}\text { E. faecalis } \\
\text { CECT7121/Ova }\end{array}$ & S/Ova \\
\hline $\begin{array}{l}\text { anti-Ova IgE } \\
\text { (OD450nm) }\end{array}$ & & $0.553 \pm 0.277$ & $0.629 \pm 0.367$ \\
\hline $\begin{array}{c}\text { anti-Ova IgG } \\
\text { (titre) }\end{array}$ & & $108.988 \pm 15.563$ & $93.561 \pm 12.610$ \\
\hline $\begin{array}{l}\text { anti-Ova IgG1 } \\
\text { (titre) }\end{array}$ & & $67.057 \pm 31.528$ & $59.452 \pm 34.763$ \\
\hline $\begin{array}{l}\text { anti-Ova IgG2a } \\
\text { (titre) }\end{array}$ & & $5425 \pm 2315^{\star}$ & $7835 \pm 3709$ \\
\hline anti-Ova & & & \\
\hline $\begin{array}{c}\text { IgG1/anti-Ova } \\
\text { IgG2a }\end{array}$ & & $12.70 \pm 4.88^{*}$ & $8.26 \pm 4.08$ \\
\hline
\end{tabular}

Antibody titres were defined as the mean inversion of dilution at which the absorbance value is 0.5 of each mouse. Data represent means \pm SEM (n:12). ${ }^{\star} \mathrm{P}<0.05$, Student's t-test. 


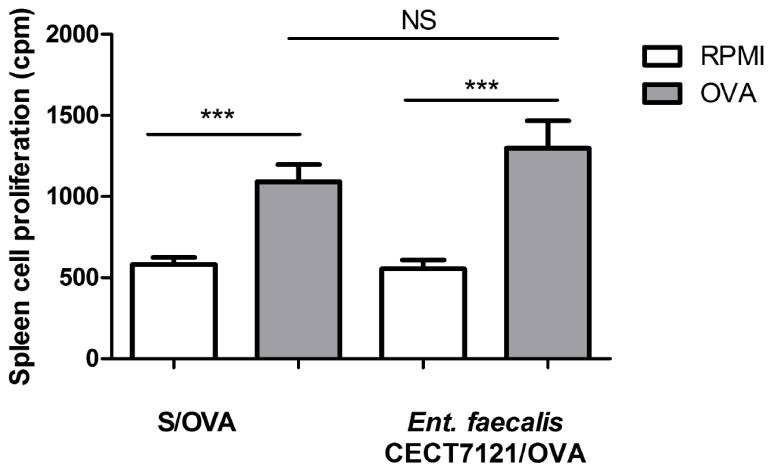

Figure 2. Comparative cell responses. On day 34 after first immunization, spleen cells of each immunized mouse were isolated and incubated with culture medium (RPMI) or OVA. Five mice from each group were analyzed in each assay and results expressed as mean count per minute (cpm). Bars represent results of three separate assays. ${ }^{* *} \mathrm{P}<0.001$, Kruskal-Wallis followed by the Dunns multiple comparisons test”.
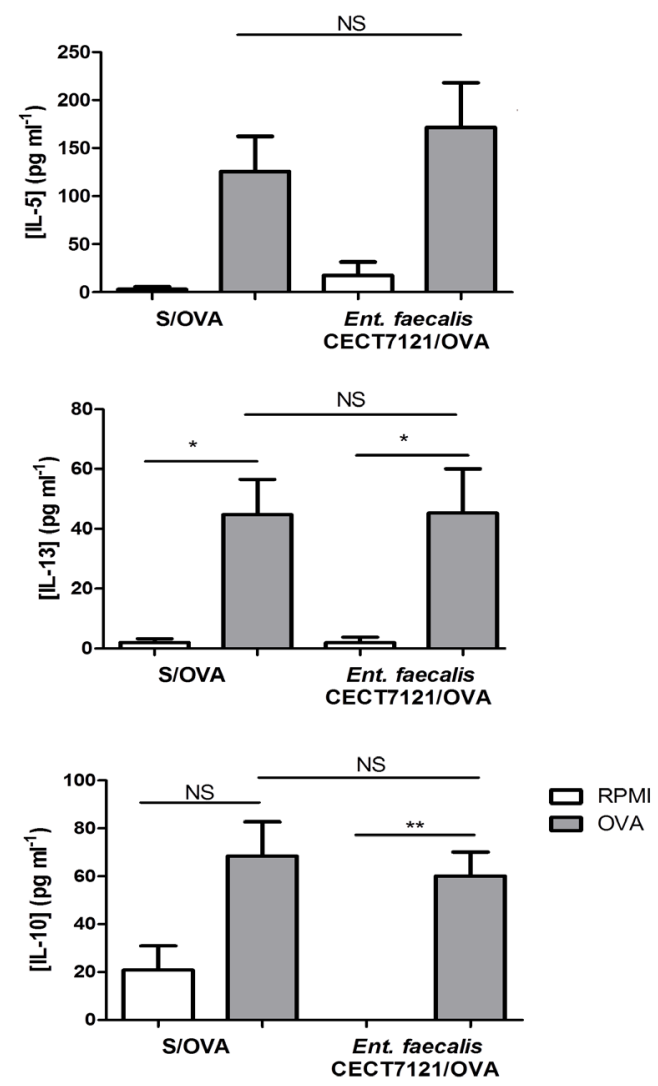

Figure 3. Th2 cytokine production. IL-5, IL-13 and IL-10 levels measured by ELISA in splenocytes. IL-5, IL-13 and IL-10 levels measured by ELISA in splenocytes culture supernatants after incubation with culture medium (RPMI) or OVA for $72 \mathrm{~h}$. Five mice from each group were analyzed in each assay and results expressed as $\mathrm{pg} \cdot \mathrm{ml}^{-1}$. Bars represent results from three separate assays. ${ }^{*} \mathrm{P}<0.01,{ }^{\star} \mathrm{P}<0.05$, Kruskal-Wallis followed by the Dunns multiple comparisons test. 


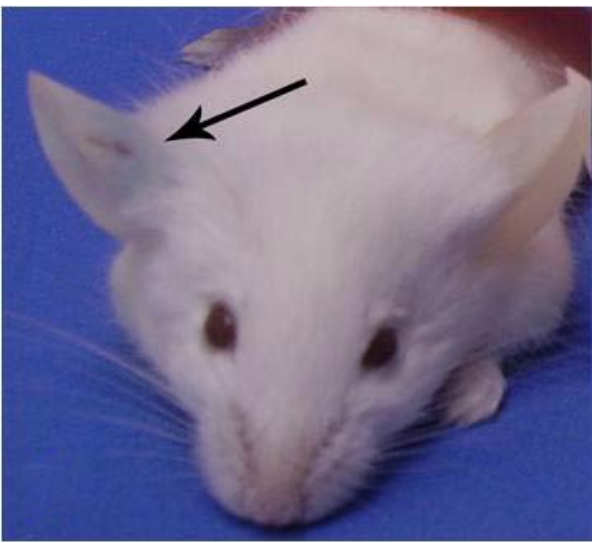

(a)

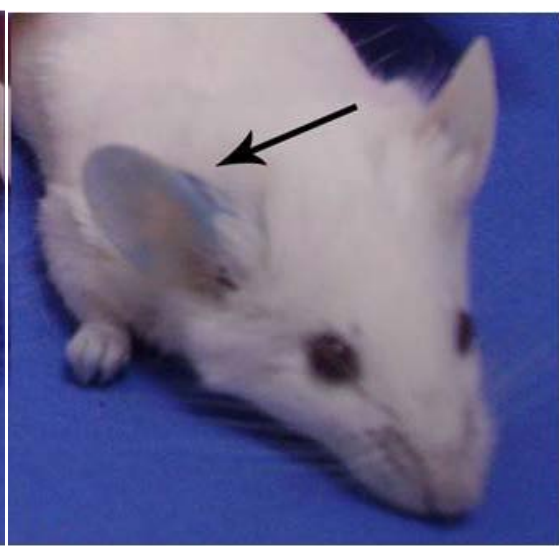

(b)

Figure 4. Active cutaneous anaphylaxis. After the intravenous injection of $1 \% \mathrm{w} / \mathrm{v}$ Evans Blue $(50 \mu \mathrm{l})$ by the tail vein, ACA was induced by intradermic challenge with the allergen in OVA-immunized, OVA/E. faecalis CECT7121 and control mice (picture not shown) in the right ear by inoculation of $50 \mu \mathrm{l}$ OVA $(1 \mathrm{mg} / \mathrm{ml})$. PBS was inoculated in the right ear of each animal as control reaction. Animals were observed after $30 \mathrm{~min}$. a) S/OVA group; b) E. faecalis CECT7121/OVA group. Arrows show the inoculation site in immunized animals.

agreement with the titres of specific IgE found in allergic animals, since it is known that IL-4, IL-5 and IL-13 are the cytokines involved in IgE synthesis regulation [2] [29] [30].

Even though the immunological ex vivo determinations indicated that $E$. faecalis CECT7121 was incapable of reverting the allergic status, the in vivo ACA test was performed, since it has been demonstrated that this test can account for amelioration of the allergic symptoms, as is the case of the treatment with Lactococcus lactis NCC2287, even when a decrease in the levels of specific antibodies is not observed [31]. In the ACA test carried out in our model, those animals belonging to the $E$. faecalis CECT7121/OVA group displayed the same degree of dye extravasation as control S/OVA animals (Figure 4), evidencing vasodilatation and a vascular permeability increase caused by mast cells degranulation.

Taking into account the results obtained herein, together with those published demonstrating the preventive effect of $E$. faecalis CECT7121 on the development of allergy [23], it can be concluded that this bacterium as it was administered could decrease the anti-OVA IgG1/IgG2a ratios observed in the group of animals treated with the bacterium due to a rise in the levels of specific IgG2a, but it could not reduce IgE levels nor show differences in the ACA test, so it would not be appropriate for the treatment of allergic symptoms in the OVA allergy induced model. These results reinforce the hypothesis that each probiotic strain can have a differential effect on both phases of the allergic response.

\section{Acknowledgements}

This study was supported by grants from Universidad de Buenos Aires (Argentina) and Fundación Alberto J. Roemmers. 


\section{References}

[1] Wang, C.C. and Rook, G.A.W. (1998) Inhibition of an Established Response to Ovalbumin in BALB/c Mice by Killed Mycobacterium vaccae. Journal of Immunology, 93, 307-313. https://doi.org/10.1046/j.1365-2567.1998.00432.x

[2] Crestani, E., Lohman, C.I., Guerra, S., Wright, A.L. and Halonen, M. (2007) Association of IL-5 Cytokine Production and in vivo IgE Levels in Infants and Parents. Journal of Allergy and Clinical Immunology, 120, 820-826.

https://doi.org/10.1016/j.jaci.2007.05.033

[3] Peng, S., Lin, J.Y. and Lin, M.Y. (2007) Antiallergic Effect of Milk Fermented with Lactic Acid Bacteria in a Murine Animal Model. Journal of Agricultural and Food Chemistry, 55, 5092-5096. https://doi.org/10.1021/jf062869s

[4] Nonaka, Y., Izumo, T., Izumi, F., Maekawa, T., Shibata, H., Nakano, A., Kishi, A., Akatani, K. and Kiso, Y. (2008) Antiallergic Effects of Lactobacillus pentosus Strain S-PT84 Mediated by Modulations of Th1/Th2 Immunobalance and Inductions of IL-10 Production. International Archives of Allergy and Immunology, 145, 249-257. https://doi.org/10.1159/000109294

[5] Michail, S. (2009) The Role of Probiotics in Allergic Diseases. Allergy, Asthma \& Clinical Immunology, 5, 5-11. https://doi.org/10.1186/1710-1492-5-5

[6] Imada, M., Estelle, F., Simons, R., Jay, F.T. and Hayglass, K.T. (1995) Allergen-Stimulated Interleukin-4 and Interferon-y Production in Primary Culture: Responses of Subjects with Allergic Rhinitis and Normal Controls. Journal of Immunology, 85, 373-380.

[7] Kim, H., Kwack, K., Kim, D.Y. and Ji, G.E. (2005) Oral Probiotic Bacterial Administration Suppressed Allergic Responses in an Ovalbumin-Induced Allergy Mouse Model. FEMS Immunology \& Medical Microbiology, 45, 259-267.

https://doi.org/10.1016/j.femsim.2005.05.005

[8] Guarner, F., Bourdet-Sicard, R., Brandtzaeg, P., Gill, H.S., McGuirk, P., van Eden, W., Versalovic, J., Weinstock, J.V. and Rook, G.A.W. (2006) Mechanisms of Disease: The Hygiene Hypothesis Revisited. Nature Clinical Practice Gastroenterology \& Hepatology, 3, 275-284. https://doi.org/10.1038/ncpgasthep0471

[9] Yeun, K.J., Choi, Y.O. and Ji, G.E. (2008) Effect of Oral Probiotics (Bifidobacterium lactis AD011 and Lactobacillus acidophilus AD031) Administration on Ovalbumin-Induced Food Allergy Mouse Model. Journal of Microbiology and Biotechnology, 18, 1393-1400.

[10] Maldonado-Galdeano, C.M., de Moreno de Le Blanc, A., Vinderola, G., Bonet, M.E. and Perdigón, G. (2007) Proposed Model: Mechanisms of Immunomodulation Induced by Probiotic Bacteria. Clinical and Vaccine Immunology, 14, 485-492. https://doi.org/10.1128/CVI.00406-06

[11] Velez, E.M., Maldonado Galdeano, C., Carmuega, E., Weill, R., Bibas Bonet, M.E. and Perdigón, G. (2011) Probiotic Fermented Milk Consumption Modulates the Allergic Process Induced by Ovoalbumin in Mice. British Journal of Nutrition, 110, 500-508.

[12] Schiavi, E., Barletta, B., Butteroni, C., Corinti, S., Boirivant, M. and Di Felice, G. (2011) Oral Therapeutic Administration of a Probiotic Mixture Suppresses Established Th2 Responses and Systemic Anaphylaxis in a Murine Model of Food Allergy. Allergy, 66, 499-508. https://doi.org/10.1111/j.1398-9995.2010.02501.x

[13] Maldonado-Galdeano, C., Novotny Núñez, I., de Moreno de LeBlanc, A., Carmuega, E., Weill, R. and Perdigón, G. (2011) Impact of a Probiotic Fermented Milk in the 
Gut Ecosystem and in the Systemic Immunity Using a Non-Severe Protein-EnergyMalnutrition Model in Mice. BMC Gastroenterology, 11, 64-78. https://doi.org/10.1186/1471-230X-11-64

[14] Zhang, J., Su, H., Li, Q., Wu, H., Liu, M., Huang, J., Zeng, M., Zheng, Y. and Sun, X. (2017) Oral Administration of Clostridium butyricum CGMCC0313-1 Inhibits $\beta$-Lactoglobulin-Induced Intestinal Anaphylaxis in a Mouse Model of Food Allergy. Gut Pathology, 9, 11-21. https://doi.org/10.1186/s13099-017-0160-6

[15] Yang, B., Xiao, L., Liu, S., Liu, Y., Luo, Y., Ji, Q., Yang, P. and Liu, Z. (2017) Exploration of the Effect of Probiotics Supplementation on Intestinal Microbiota of Food Allergic Mice. American Journal of Translational Research, 9, 376-385.

[16] Moreno, M.F., Sarantinopoulos, P., Tsakalidou, E. and De Vuyst, L. (2006) The Role and Application of Enterococci in Food and Health. International Journal of Food Microbiology, 106, 1-24. https://doi.org/10.1016/j.ijfoodmicro.2005.06.026

[17] Franz, C.M., Huch, M., Abriouel, H., Holzapfel, W. and Gálvez, A. (2011) Enterococci as Probiotics and Their Implications in Food Safety. International Journal of Food Microbiology, 151, 125-140. https://doi.org/10.1016/j.ijfoodmicro.2011.08.014

[18] Sparo, M., Nuñez, G.G., Castro, M., Calcagno, M.L., García Allende, M.A., Ceci, M., Najle, R. and Manghi, M. (2008) Characteristics of an Environmental Strain, Enterococcus faecalis CECT7121, and Its Effects as Additive on Craft Dry-Fermented Sausages. Food Microbiology, 25, 607-615. https://doi.org/10.1016/j.fm.2008.01.008

[19] Castro, M., Sparo, M., Molina, M., Andino, J. and Manghi, M. (2007) Enterococcus faecalis CECT7121 Induces Systemic Immunomodulatry Effects and Protects from Salmonella Infection. International Journal of Probiotics and Prebiotics, 2, 215-224.

[20] Castro, M.S., Molina, M.A., Azpiroz, M.B., Díaz, A.M., Ponzio, R., Sparo, M.D., Manghi, M.A. and Canellada, A.M. (2016) Probiotic Activity of Enterococcus faecalis CECT7121: Effects on Mucosal Immunity and Intestinal Epithelial Cells. Journal of Applied Microbiology, 121, 1117-1129. https://doi.org/10.1111/jam.13226

[21] Castro, M., Molina, M., Sparo, M. and Manghi, M. (2008) Effects of Enterococcus faecalis CECT7121 on the Specific Immune Response after DTPw Vaccination. International Journal of Probiotics and Prebiotics, 3, 25-30.

[22] Castro, M.S., Molina, M.A., Di Sciullo, P., Azpiroz, M.B., Leocata Nieto, F., Sterín-Speziale, N.B., Mongini, C. and Manghi, M.A. (2010) Beneficial Activity of Enterococcus faecalis CECT7121 in the Anti-Lymphoma Protective Response. Journal of Applied Microbiology, 109, 1234-1243. https://doi.org/10.1111/j.1365-2672.2010.04747.x

[23] Castro, M.S., Azpiroz, M.B., Molina, M.A., Mourelle, A.C., Alaniz, F.S., Maldonado, A.M. and Manghi, M.A. (2012) Preliminary Studies on the Prevention of the Ovalbumin-Induced Allergic Response by Enterococcus faecalis CECT7121 in Mice. International Archives of Allergy and Immunology, 157, 11-20. https://doi.org/10.1159/000324673

[24] Matsuzaki, T., Yamazaki, R., Hashimoto, S. and Yokokura, T. (1997) Antidiabetic Effect of an Oral Administration of Lactobacillus casei in a Non-Insulin Dependent Diabetes Mellitus (NIDDM) Model Using KK-Ay Mice. Endocrine Journal, 44, 357-365. https://doi.org/10.1507/endocrj.44.357

[25] Kalliomäki, M., Salminen, S., Arvilommi, H., Kero, P., Koskinen, P. and Isolauri, E. (2001) Probiotics in Primary Prevention of Atopic Disease: A Randomized Placebo-Controlled Trial. Lancet, 357, 1076-1079. https://doi.org/10.1016/S0140-6736(00)04259-8

[26] Ishida, Y., Bandou, I., Kanzato, H. and Yamamoto, N. (2003) Decrease in Ovalbu- 
min Specific IgE of Mice Serum after Oral Uptake of Lactic Acid Bacteria. Bioscience, Biotechnology, and Biochemistry, 67, 951-957.

https://doi.org/10.1271/bbb.67.951

[27] Hougee, S., Vriesema, A.J.M., Wijering, S.C., Knippels, L.M.J., Folkerts, G., Nijkamp, F.P., Knol, J. and Garssen, J. (2010) Oral Treatment with Probiotics Reduces Allergic Symptoms in Ovalbumin Sensitized Mice: A Bacterial Strain Comparative Study. International Archives of Allergy and Immunology, 151, 107-117. https://doi.org/10.1159/000236000

[28] Walker, W.A. (2014) Intestinal Colonization and Programming of the Intestinal Immune Response. Journal of Clinical Gastroenterology, 4813, S8-S11. https://doi.org/10.1097/MCG.0000000000000230

[29] Gould, H.J., Sutton, B.J., Beavil, A.J., Beavil, R.L., McCloskey, N., Coker, H.A., Fear, D. and Smurthwaite, L. (2003) The Biology of IgE and the Basis of Allergic Disease. Annual Review of Immunology, 21, 579-628.

https://doi.org/10.1146/annurev.immunol.21.120601.141103

[30] Levine, S.J., and Wenzel, S.E. (2010) Narrative Review: The Role of Th2 Immune Pathway Modulation in the Treatment of Severe Asthma and Its Phenotypes. Annals of Internal Medicine, 152, 232-237. https://doi.org/10.7326/0003-4819-152-4-201002160-00008

[31] Zuercher, A.W., Weiss, M., Holvoet, S., Moser, M., Moussu, H., van Overtvelt, L., Horiot, S., Moingeon, P., Nutten, S., Prioult, G., Singh A. and Mercenier, A. (2012) Lactococcus lactis NCC 2287 Alleviates Food Allergic Manifestations in Sensitized Mice by Reducing IL-13 Expression Specifically in the Ileum. Clinical \& Develop mental Immunology, 2012, Article ID: 485750. https://doi.org/10.1155/2012/485750 